\title{
Multiscale Models with Moisture and Systematic Strategies for Superparameterization
}

\author{
ANDREW J. MAJDA \\ Department of Mathematics, and Center for Atmosphere/Ocean Science, Courant Institute, New York University, \\ New York, New York
}

(Manuscript received 29 June 2006, in final form 9 November 2006)

\begin{abstract}
The accurate parameterization of moist convection presents a major challenge for the accurate prediction of weather and climate through numerical models. Superparameterization is a promising recent alternative strategy for including the effects of moist convection through explicit turbulent fluxes calculated from a cloud-resolving model. Basic scales for cloud-resolving modeling are the microscales on the order of $10 \mathrm{~km}$ in space on time scales on the order of $15 \mathrm{~min}$, where vertical and horizontal motions are comparable and moist processes are strongly nonlinear (meso-gamma scale). In this paper, systematic multiscale asymptotic analysis is utilized to develop simplified microscale mesoscale dynamic (MMD) models for interaction between the microscales and spatiotemporal mesoscales on the order of $100 \mathrm{~km}$ and $2.5 \mathrm{~h}$ (meso-beta scale). The new MMD models lead to a systematic framework for superparameterization for numerical weather prediction (NWP) generalizing the traditional column modeling framework. The MMD formulation also provides a flexible systematic framework for devising new parameterization strategies for NWP intermediate between the two extremes of column modeling and detailed cloud-resolving modeling. It is also established here that these MMD models fit crudely into the recent systematic multiscale framework developed to explain the observed larger-scale statistical self-similarity of tropical convection, and therefore provide a systematic framework for superparameterization. Finally, it is shown that the new MMD models have the structure of a heterogeneous multiscale method so that many numerical techniques recently developed in the applied mathematics literature can be applied to this formulation.
\end{abstract}

\section{Introduction}

A major stumbling block in the accurate prediction of weather and short-term climate is the accurate parameterization of moist convection in numerical models (Emanuel 1994; Moncrieff and Liu 2006). Recently, an alternative strategy for including the effects of moist convection in numerical models through explicit turbulent fluxes calculated from a cloud-resolving model has been developed called superparameterization (Grabowski 2001, 2003, 2004; Randall et al. 2003). Superparameterization blends conventional parameterization on a coarse mesh with detailed cloud-resolving modeling on a finer mesh with an imposed scale gap. This method has yielded promising new results regarding tropical intraseasonal behavior (Grabowski 2001, 2003, 2004; Khairoutdinov et al. 2005), but a systematic for-

Corresponding author address: Prof. Andrew J. Majda, Courant Institute, New York University, 251 Mercer Street, New York, NY 10012.

E-mail: jonjon@cims.nyu.edu

DOI: $10.1175 / J A S 3976.1$

(C) 2007 American Meteorological Society mulation and analysis for such superparameterization strategies that might lead to algorithmic improvements are not yet available despite interesting progress in this direction (Grabowski 2004, 2006; Grabowski and Moncrieff 2004; Jung and Arakawa 2005). One of the major stumbling blocks for general circulation models is their poor capability in resolving convectively coupled tropical synoptic-scale waves and intraseasonal oscillations (Slingo et al. 1996; Lin et al. 2006). One conjectured reason for this poor performance of contemporary numerical models is their inadequate representation of interactions across multiple spatiotemporal scales in the Tropics. Recent processing of observational data (Mapes et al. 2006) suggests the statistical self-similarity of tropical convection from the smallest, shortest scales to organized mesoscale convective systems (Houze 2004) to convective clusters to equatorial synoptic-scale superclusters to planetary/intraseasonal oscillations. For this reason, it is interesting to develop systematic multiscale asymptotic models (Klein 2000, 2004; Majda 2003; Majda and Klein 2003) for the nonlinear cascade across scales in the Tropics, and the author has done 
this recently for the self-similar behavior from mesoscales to planetary/intraseasonal scales (Majda 2007). Such quantitative models are useful for quantifying the observed multiscale behavior in, for example, tropical intraseasonal oscillations (Majda and Biello 2004; Biello and Majda 2005, 2006).

Important spatiotemporal scales for cloud-resolving modeling are the fully nonlinear scales with active moisture, called the microscales here, involving spatiotemporal scales

$$
L_{m}=10 \mathrm{~km} \text { and } T_{m}=15 \mathrm{~min},
$$

where strong comparable vertical and horizontal velocities on the order of $10 \mathrm{~m} \mathrm{~s}^{-1}$ together with fully nonlinear bulk cloud microphysics are allowed (Lipps and Hemler 1982; Klein and Majda 2006). In conventional terminology (Orlanski 1975) these are the meso- $\gamma$ scales, but the viewpoint here is that (1.1) represents the integral scale for a numerical method resolving microscale deep convective towers (Orlanski 1975); this motivates the microscale terminology used here. The organized impact of moist convection on the scales in (1.1) through squall lines and other mesoscale convective systems (Moncrieff 1992; Emanuel 1994; Pandya and Durran 1996; Houze 2004) occurs on the mesoscale spatiotemporal scales, $L_{M}$ and $T_{M}$, with

$$
L_{M}=100 \mathrm{~km}=\epsilon^{-1} L_{m} \quad \text { and } \quad T_{M}=2.5 \mathrm{~h}=\epsilon^{-1} T_{m}
$$

for $\epsilon \approx 0.1$. The mesoscales in (1.2) are the meso- $\beta$ scales in conventional terminology (Orlanski 1975). In (1.2) and elsewhere in the paper, hard numbers are identified with an asymptotic statement valid for $\epsilon \ll 1$ by using the conservative choice $\epsilon \approx 0.1$ [see Majda and Klein (2003) and Biello and Majda (2005) for a similar use of these conventions]. Contemporary numerical weather prediction (NWP) models (Moncrieff and Liu 2006) currently can have a mesh size on the order of 10 $\mathrm{km}=L_{m}$, so new numerical strategies that lead to the improved representation of the mesoscale impacts of tropical convection have central importance. It is also interesting to see how the strongly nonlinear scales with active moisture in (1.1) and (1.2) feed into the nonlinear multiscale cascade creating the observed statistical self-similarity of tropical convection (Mapes et al. 2006; Majda 2007).

The present paper addresses these issues through systematic multiscale modeling (Klein 2000, 2004; Majda 2003; Klein and Majda 2006) for the spatiotemporal scales in (1.1) and (1.2). After a preliminary section on the moist anelastic equations, the main results in the paper are presented in section 3; there it is shown that

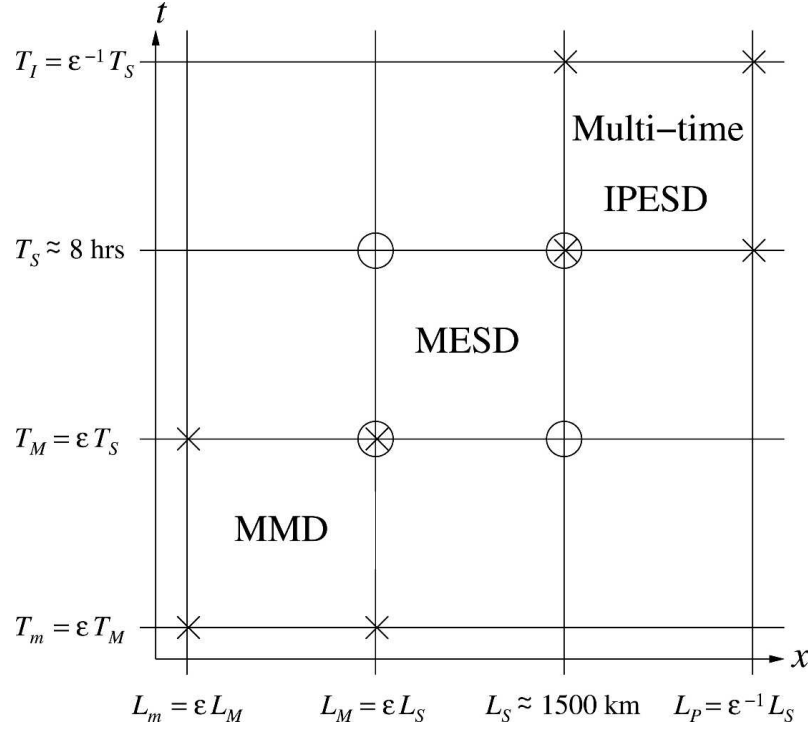

FIG. 1. The multiple spatiotemporal scales spanned here with the MMD and MESD models with overlap on the mesoscales and the MESD and multitime intraseasonal planetary equatorial synoptic dynamics (IPESD) models (Majda 2007) with overlap on equatorial synoptic scales.

the systematic multiscale analysis naturally leads to new simplified equations for microscale mesoscale dynamics (MMD) that yield a new systematic superparameterization strategy for NWP. In section 4, a crude analytic link is established between the fully nonlinear MMD models and the other larger-scale multiscale models (Majda 2007) for the nonlinear cascade in the Tropics across all spatiotemporal scales from microscales to planetary/intraseasonal (see Fig. 1); this yields further analytic insight for the observed statistical selfsimilarity of tropical convection (Mapes et al. 2006) across all these scales; these results also give insight for a systematic framework for the original superparameterization strategy (Grabowski 2004). In section 5 numerical issues for the implementation of the systematic strategy for superparameterization are discussed briefly. In particular, in section $5 \mathrm{a}$ it is noted that the formulation for NWP superparameterization from section 3 can be viewed as a heterogeneous multiscale method (HMM; E and Engquist 2003); thus, many numerical techniques recently developed in the applied mathematical literature can be utilized for this new systematic superparameterization strategy. The HMM formulation is a systematic development of multiscale numerical parameterization strategies that generalizes earlier work in diverse disciplines [see Schmidt and Klein (2003) and the numerous references in the introduction from Majda et al. (2005) for other examples]. In section $5 \mathrm{~b}$, there is a brief discussion of the potential 
use of the present flexible systematic formulation in designing new strategies for NWP that retain the fidelity of cloud-resolving modeling with roughly $1-\mathrm{km}$ resolution and significantly reduced computational overhead.

\section{Preliminaries}

Here the moist nonhydrostatic anelastic equations with bulk cloud microphysics (Lipps and Hemler 1982; Grabowski and Smolarkiewicz 1996) are utilized as the basic equations for the microscale dynamics. With suitable nondimensional units explained below (Klein and Majda 2006), which have the microscale space-time scales

$$
L_{m}=10 \mathrm{~km} \text { and } T_{m}=15 \mathrm{~min},
$$

these equations are given by

$$
\begin{aligned}
\frac{D \mathbf{u}_{h}}{D t}+\epsilon^{4} y \mathbf{u}_{h}^{\perp}+\epsilon \mathbf{i} w & =-\nabla_{h} p \\
\frac{D w}{D t}-\epsilon u & =-p_{z}+\theta+\left(\bar{\epsilon} q_{v}-q_{r}-q_{c}\right) \\
\frac{D \theta}{D t}+N^{2}(z) w & =L \frac{\theta_{0}}{p_{0}}\left(C_{d}-E_{r}\right) \\
\operatorname{div}_{h} \mathbf{u}_{h}+\rho^{-1}(\rho w)_{z} & =0
\end{aligned}
$$

with

$$
\frac{D}{D t}=\frac{\partial}{\partial t}+\mathbf{u}_{h} \cdot \nabla_{h}+w \frac{\partial}{\partial z}
$$

and

$$
\begin{aligned}
\frac{D q_{v}}{D t} & =-C_{d}+E_{r} \\
\frac{D q_{c}}{D t} & =C_{d}-A_{r} \\
\frac{D q_{r}}{D t}-\frac{1}{\rho} \frac{\partial}{\partial z}\left(\rho V_{T} q_{r}\right) & =A_{r}-E_{r} .
\end{aligned}
$$

In (2.2), the units for both horizontal, $\mathbf{u}_{h}=(u, v)$, and vertical, $w$, velocity are $10 \mathrm{~m} \mathrm{~s}^{-1}$ while $\rho(z), N^{2}(z)$, $\theta_{0}(z)$, and $\rho_{0}(z)$ are the nondimensional forms of the dry statically stable vertical profile with the equatorial $\beta$-plane approximation for the Coriolis terms (Majda and Klein 2003; Klein and Majda 2006). Here $\bar{\epsilon}+1$ is the ratio of gas constants of water vapor and dry air, and $p_{\text {ref }}=10^{5} \mathrm{~kg} \mathrm{~m}^{-2}$ and $\rho_{\text {ref }}=1 \mathrm{~kg} \mathrm{~m}^{-3}$. The potential temperature in (2.2) is scaled according to the dry lapse rate; that is, the units of $\theta$ are $30 \mathrm{~K}$ over a height of $10 \mathrm{~km}$, which is comparable with the latent heating/ cooling due to moist processes [see Klein and Majda (2006), their Eqs. (39)-(42)]. The quantities $q_{v}, q_{c}$, and $q_{r}$ are the mixing ratios for cloud vapor, water, and rain, respectively, rescaled by the factor $\epsilon^{-2}$. This rescaling renders the potential temperature equation in (2.2) dimensionless with order-1 latent heat prefactor $L$ and simultaneously guarantees that the condensation of cloud vapor, $C_{d}$, evaporation of rain, $E_{r}$, and the conversion of cloud water to rain by both autoconversion and collection, $A_{r}$, as well as the fall velocity, $V_{T}$, are order one processes on the time scales in (2.1) (Klein and Majda 2006). While the detailed forms of $C_{d}, E_{r}$, and $A_{r}$ (Emanuel 1994; Grabowski and Smolarkiewicz 1996) are not needed for the discussion here, the tacit standard assumption utilized in achieving a single time scale governing the moisture source terms in (2.2) and (2.3) is that the very fast processes associated with supersaturated water vapor are equilibrated by constraining the cloud vapor to always lie below or equal to saturation (Lipps and Hemler 1982; Grabowski and Smolarkiewicz 1996; Klein and Majda 2006). For simplicity in exposition, all other source terms and dissipation in (2.2) and (2.3) have been set to zero; they are readily added in the analysis below. The equations in (2.2) and (2.3) have been utilized extensively as the microscale dynamical equations in both cloud-resolving modeling and superparameterization (Grabowski and Moncrieff 2001; Grabowski 2001, 2003, 2004), so they are a natural starting point for this paper. The analysis below also applies to the moist compressible Euler equations as the microscopic model (Klein 2000; Klein and Majda 2006) with a more cumbersome and lengthy but similar asymptotic argument required. Actually, recent detailed analysis establishes that there is subtle behavior in general in the anelastic limit at low Mach numbers for a compressible atmosphere (Klein 2000, 2004), but this issue is not discussed further here.

\section{Multiple-scale asymptotics}

Here the focus is on systematic multispace-multitime-scale asymptotic solutions of (2.2) and (2.3) on the microscales in (2.1) and mesoscales, $L_{m}$ and $T_{m}$, with

$$
\begin{aligned}
& L_{M}=\epsilon^{-1} L_{m}=O(100 \mathrm{~km}) \quad \text { and } \\
& T_{M}=\epsilon^{-1} T_{m}=O(2 \mathrm{~h}) .
\end{aligned}
$$

With (2.1) and (2.4) as the essential spatiotemporal scales, general asymptotic solutions of (2.2) and (2.3) are developed with the form

$$
\begin{aligned}
\mathbf{u}_{h} & =\left[\overline{\mathbf{u}}_{h}(\epsilon \mathbf{x}, z, \epsilon t, t)+\epsilon \overline{\mathbf{u}}_{h, 1}\right]+\mathbf{u}_{h}^{\prime}(\epsilon \mathbf{x}, \mathbf{x}, z, \epsilon t, t)+O\left(\epsilon^{2}\right) \\
w & =\left(\bar{w}+\epsilon \bar{w}_{1}\right)+w^{\prime}+O\left(\epsilon^{2}\right) \\
\theta & =\left(\bar{\theta}+\epsilon \bar{\theta}_{1}\right)+\theta^{\prime}+O\left(\epsilon^{2}\right) \\
p & =\left(\bar{p}+\epsilon \bar{p}_{1}\right)+p^{\prime}+O\left(\epsilon^{2}\right) \\
\mathbf{q} & =\left(\overline{\mathbf{q}}+\epsilon \overline{\mathbf{q}}_{1}\right)+\mathbf{q}^{\prime}+O\left(\epsilon^{2}\right),
\end{aligned}
$$


where $\mathbf{q}=\left(q_{v}, q_{c}, q_{r}\right)$. In (2.5) the larger-scale longer time variables,

$$
\mathbf{X}=\epsilon \mathbf{X} \quad \text { and } \quad T=\epsilon t,
$$

account for mesoscale modulations. In accordance with conventional multiple-scale asymptotic analysis (Klein 2000; Majda 2003, 2007; Klein and Majda 2006), given a general function $f(\epsilon \mathbf{x}, \mathbf{x}, \epsilon t, t)$, the spatial and time averages of $f$ over the microscales are given, respectively, by

$$
\begin{aligned}
\bar{f}(\mathbf{X}, T, t) & =\lim _{L \rightarrow \infty} \frac{1}{(2 L)^{2}} \int_{-L}^{L} \int_{-L}^{L} f(\mathbf{X}, \mathbf{x}, T, t) d x d y \\
\langle f\rangle(\mathbf{X}, \mathbf{x}, T) & =\lim _{\tilde{T} \rightarrow \infty} \frac{1}{2 \tilde{T}} \int_{-\tilde{T}}^{\tilde{T}} f(\mathbf{X}, \mathbf{x}, T, t) d t
\end{aligned}
$$

with $(X, T)$ and $(x, t)$ regarded as independent variables in (2.7). With the first equation in (2.7), a function $f$ admits the decomposition, $f=\bar{f}+f^{\prime}$ with $\bar{f}^{\prime}=0$, which gives a conventional separation of the terms in (2.5) into their spatial means and fluctuations. Note that the spatiotemporal average involves variations only on the larger, longer mesoscales in (2.4), that is,

$$
\langle\bar{f}\rangle(\mathbf{X}, z, T) .
$$

In the next section, systematic asymptotic equations are developed for the mesoscale averages, $\left\langle\overline{\mathbf{u}}_{h}\right\rangle(\mathbf{X}, z, T)$, etc. from (2.2) and (2.3), which are the quantities of interest. In the multiscale procedure utilized in the next section, the space-time gradient of $f(\epsilon \mathbf{x}, \mathbf{x}, \epsilon t, t)$ is calculated according to the chain rule as

$$
\epsilon \boldsymbol{\nabla}_{X} f+\nabla^{\prime} f, \quad \epsilon \frac{\partial f}{\partial T}+\frac{\partial f}{\partial t},
$$

where the notation $\boldsymbol{\nabla}^{\prime} f=(\partial f / \partial x, \partial f / \partial y)$ denotes the horizontal small-scale gradient of the fluctuations.

\section{Derivation of the microscale mesoscale dynamic model}

To develop the systematic asymptotic model, the ansatz in (2.5) is substituted into (2.2) and (2.3), and leading-order equations for the mean and spatial fluctuations are developed on the microscale time.

\section{a. Column model dynamics on spatial mesoscales}

First, the spatial average of the mass conservation equation is given by

$$
\rho^{-1}(\rho \bar{w})_{z}+\epsilon\left[\operatorname{div}_{X} \overline{\mathbf{u}}_{h}+\rho^{-1}\left(\rho \bar{w}_{1}\right)_{z}\right]=0 .
$$

The only way to solve (3.1) at leading order is to require

$$
\bar{w}(\mathbf{X}, z, \epsilon t, t) \equiv 0
$$

Thus, the first nontrivial vertical velocity average on mesoscales is given by $\epsilon \bar{w}_{1}$, which involves magnitudes of roughly $1 \mathrm{~m} \mathrm{~s}^{-1}$. The constraint in (3.2) was imposed on an ad hoc basis in superparameterization (Grabowski 2004); here this constraint is derived systematically from (3.1). With (3.1) and (3.2), the remaining equations of order one for the mesoscale spatial mean on microtime scales are given by the column model equations,

$$
\begin{aligned}
& \bar{p}_{z}=\bar{\theta}+\bar{\epsilon} \bar{q}_{v}-\bar{q}_{r}-\rho^{-1}\left(\rho \overline{w^{\prime 2}}\right)_{z} \\
& \frac{\partial \overline{\mathbf{u}}_{h}}{\partial t}=-\rho^{-1}\left(\rho \overline{w^{\prime} \mathbf{u}_{h}^{\prime}}\right)_{z} \\
& \frac{\partial \bar{\theta}}{\partial t}=L \frac{\theta_{0}}{p_{0}}\left(\bar{C}_{d}-\bar{E}_{r}\right)-\rho^{-1}\left(\rho \overline{\theta^{\prime} w^{\prime}}\right)_{z} \\
& \frac{\partial \bar{q}_{v}}{\partial t}=-\bar{C}_{d}+\bar{E}_{r}-\rho^{-1}\left(\rho \overline{\bar{q}_{v}^{\prime} w^{\prime}}\right)_{z} \\
& \frac{\partial \bar{q}_{c}}{\partial t}=\bar{C}_{d}-\bar{A}_{r}-\rho^{-1}\left(\rho \overline{q_{c}^{\prime} w^{\prime}}\right)_{z}
\end{aligned}
$$

$\frac{\partial \bar{q}_{r}}{\partial t}-\frac{1}{\rho}\left(\frac{\partial}{\partial z}\left(\rho V_{T} \bar{q}_{r}\right)\right)=\bar{A}_{r}-\bar{E}_{r}-\rho^{-1}\left(\rho \overline{q_{r}^{\prime} w^{\prime}}\right)_{z}$.

Note that in accordance with (3.2), the mesoscale mean pressure equation is now hydrostatic. The other equations in (3.3) impose further constraints on the largescale, longer time dynamics on mesoscales alone. With the ansatz in (2.5), obviously $\mathbf{u}_{h}, \theta$, and $\mathbf{q}$ need to remain bounded in the nondimensional units on the mesoscale time scale; since

$$
\left\langle\frac{\partial f}{\partial t}\right\rangle=0
$$

for any bounded function, the equations in (3.3) yield the following averaged constraints on source terms:

$$
\begin{aligned}
& -\rho^{-1}\left(\rho\left\langle\overline{w^{\prime} \mathbf{u}_{h}^{\prime}}\right\rangle\right)_{z}=\epsilon\left\langle\overline{\mathbf{S}}_{\mathbf{u}}\right\rangle(\mathbf{X}, z, T) \\
& L \frac{\theta_{0}}{p_{0}}\left(\left\langle\bar{C}_{d}\right\rangle-\left\langle\bar{E}_{r}\right\rangle\right)-\rho^{-1}\left(\rho\left\langle\overline{\theta^{\prime} w^{\prime}}\right\rangle\right)_{z}=\epsilon\left\langle\bar{S}_{\theta}\right\rangle \\
& -\left\langle\bar{C}_{d}\right\rangle+\left\langle\bar{E}_{r}\right\rangle-\rho^{-1}\left(\rho\left\langle\overline{q_{v}^{\prime} w^{\prime}}\right\rangle\right)_{z}=\epsilon\left\langle\bar{S}_{q v}\right\rangle \\
& \left\langle\bar{C}_{d}\right\rangle-\left\langle\bar{A}_{r}\right\rangle-\rho^{-1}\left(\rho\left\langle\overline{q_{c}^{\prime} w^{\prime}}\right\rangle\right)_{z}=\epsilon\left\langle\bar{S}_{q c}\right\rangle
\end{aligned}
$$$$
\frac{1}{\rho}\left[\frac{\partial}{\partial z}\left(\rho V_{T}\left\langle\bar{q}_{r}\right\rangle\right)\right]+\left\langle\bar{A}_{r}\right\rangle-\left\langle\bar{E}_{r}\right\rangle-\rho^{-1}\left(\rho\left\langle\overline{q_{r}^{\prime} w^{\prime}}\right\rangle\right)_{z}
$$$$
=\epsilon\left\langle\bar{S}_{q r}\right\rangle
$$ 
for each vertical level $z$. The source terms on the righthand side in (3.5) are defined by these equations. These large-scale balances for spatiotemporal averages need to be satisfied in order for the asymptotic procedure to remain valid; the first equation in (3.5) is satisfied only if the space-time average from (2.8) of the vertical flux of horizontal momentum is sufficiently weak [i.e., the space-time area fraction over the deep convective updrafts/downdrafts is sufficiently small or there is sufficient spatiotemporal decorrelation (Klein and Majda 2006)]. Otherwise the source terms in (3.5) would not be small as required by the asymptotic procedure. This can be checked "on the fly" in a numerical algorithm.

\section{b. Coarse-grained mesoscale-averaged equations}

The derivation of equations for the coarse-grained mesoscale averages is illustrated by the derivation of an equation for the potential temperature, $\langle\bar{\theta}\rangle(\mathbf{X}, z, T)$. With the ansatz in (2.5) and utilizing (2.9), the terms of the order $\epsilon$ in the potential temperature equation are given by

$$
\rho \frac{\partial \bar{\theta}_{1}}{\partial t}+\rho \frac{D \bar{\theta}}{D T}+\rho N^{2} \bar{w}_{1}=-\operatorname{div}_{X}\left(\rho \overline{\mathbf{u}_{h}^{\prime} \theta^{\prime}}\right)+\rho \bar{S}_{\theta}
$$

with $(D / D T)=(\partial / \partial T)+\overline{\mathbf{u}}_{h} \cdot \nabla_{X}+\bar{w}_{1}(\partial / \partial z)$. The condition in (3.6) requires $\left\langle\partial \bar{\theta}_{1} / \partial t\right\rangle=0$ to avoid secular growth on large scales; thus (3.5) and (3.6) yield the equation

$$
\left.\rho \frac{\partial\langle\bar{\theta}\rangle}{\partial T}+\rho N^{2}\left\langle\bar{w}_{1}\right\rangle=\rho\left\langle\bar{S}_{\theta}\right\rangle-\operatorname{div}_{X}\left(\rho \overline{\mathbf{u}_{h}^{\prime} \theta^{\prime}}\right\rangle\right)-\operatorname{div}(\rho\langle\overline{\mathbf{v}} \bar{\theta}\rangle)
$$

with $\overline{\mathbf{v}}=\left(\overline{\mathbf{u}}_{h}, \bar{w}_{1}\right)$. Note that the vertical eddy flux in (3.6) is no longer explicit and is calculated through $\bar{S}_{\theta}$ from the requirement in (3.5). The same analysis for the moisture equations yields

$$
\begin{aligned}
\frac{\partial}{\partial T}\left\langle\bar{q}_{v}\right\rangle & =\left\langle\bar{S}_{q v}\right\rangle-\operatorname{div}_{X}\left\langle\overline{q_{v}^{\prime} \mathbf{u}_{h}^{\prime}}\right\rangle-\frac{1}{\rho} \operatorname{div}\left(\rho\left\langle\bar{q}_{v} \overline{\mathbf{v}}\right\rangle\right) \\
\frac{\partial}{\partial T}\left\langle\bar{q}_{c}\right\rangle & =\left\langle\bar{S}_{q c}\right\rangle-\operatorname{div}_{X}\left\langle\overline{q_{c}^{\prime} \mathbf{u}_{h}^{\prime}}\right\rangle-\frac{1}{\rho} \operatorname{div}\left(\rho\left\langle\bar{q}_{c} \overline{\mathbf{v}}\right\rangle\right) \\
\frac{\partial}{\partial T}\left\langle\bar{q}_{r}\right\rangle & =\left\langle\bar{S}_{q r}\right\rangle-\operatorname{div}_{X}\left\langle\overline{q_{r}^{\prime} \mathbf{u}_{h}^{\prime}}\right\rangle-\frac{1}{\rho} \operatorname{div}\left(\rho\left\langle\bar{q}_{r} \overline{\mathbf{v}}\right\rangle\right),
\end{aligned}
$$

while the mesoscale horizontal momentum equation becomes

$$
\frac{\partial}{\partial T}\left\langle\overline{\mathbf{u}}_{h}\right\rangle=-\nabla_{h}\langle\bar{p}\rangle+\left\langle\overline{\mathbf{S}}_{\mathbf{u}}\right\rangle-\operatorname{div}_{X}\left\langle\overline{\overline{\mathbf{u}}_{h}^{\prime}: \mathbf{u}_{h}^{\prime}}\right\rangle-\frac{1}{\rho} \operatorname{div}\left\langle\rho \overline{\mathbf{v}}: \overline{\mathbf{u}}_{h}\right\rangle,
$$

with $\left(\rho \mathbf{v}: \mathbf{u}_{h}\right)_{i}=\rho \mathbf{v} u_{h, i}$ for $i=1,2$. The equations in (3.7)-(3.9) together with the hydrostatic balance from (3.3) and the anelastic constraint

$$
\left.\langle\bar{p}\rangle_{z}=\langle\bar{\theta}\rangle+\bar{\epsilon}\left\langle\bar{q}_{v}\right\rangle-\left\langle\bar{q}_{r}\right\rangle-\rho^{-1}\left(\rho \overline{\left\langle w^{\prime 2}\right.}\right\rangle\right)_{z}
$$

$\operatorname{div}_{X}\left\langle\overline{\mathbf{u}}_{h}\right\rangle+\rho^{-1}\left(\rho\left\langle\bar{w}_{1}\right\rangle\right)_{z}$

$$
=0
$$

complete the reduced coarse-grained dynamics on mesoscales under the necessary constraints in (3.5).

\section{c. Equations for microscale fluctuations}

To derive the equations for the microscale fluctuations, it is convenient to introduce the advection operator

$$
\frac{D^{\prime}}{D t^{\prime}}=\frac{\partial}{\partial t}+\left(\overline{\mathbf{u}}_{h}+\mathbf{u}_{h}^{\prime}\right) \cdot \nabla_{h}^{\prime}+w^{\prime} \frac{\partial}{\partial z},
$$

which includes the mesoscale mean calculated from (3.3). With (3.3) and inserting the ansatz from (2.5) into (2.2), the bounded leading-order terms satisfy the equations for microscale fluctuations:

$$
\begin{aligned}
\frac{D^{\prime} \mathbf{u}_{h}^{\prime}}{D t^{\prime}}= & -\nabla_{h}^{\prime} p^{\prime}-w^{\prime} \frac{\partial \overline{\mathbf{u}}_{h}}{\partial z}+\rho^{-1}\left(\rho \overline{\mathbf{u}_{h}^{\prime} w^{\prime}}\right)_{z} \\
\frac{D^{\prime} w^{\prime}}{D t^{\prime}}= & -p_{z}^{\prime}+\theta^{\prime}+\left(\bar{\epsilon} q_{v}^{\prime}-q_{c}^{\prime}-q_{r}^{\prime}\right) \\
& +\rho^{-1}\left(\overline{\rho w^{\prime 2}}\right)_{z} \\
\frac{D^{\prime} \theta^{\prime}}{D t^{\prime}}+N^{2} w^{\prime}= & L \frac{\theta_{0}}{p_{0}}\left(C_{d}^{\prime}-E_{r}^{\prime}\right)-w^{\prime} \frac{\partial \bar{\theta}}{\partial z} \\
& +\rho^{-1}\left(\rho \overline{\theta^{\prime} w^{\prime}}\right)_{z}
\end{aligned}
$$

$\operatorname{div}^{\prime} \mathbf{u}_{h}^{\prime}+\rho^{-1}\left(\rho w^{\prime}\right)_{z}=0$

$$
\begin{gathered}
\frac{D^{\prime} q_{v}^{\prime}}{D t^{\prime}}=-C_{d}^{\prime}+E_{r}^{\prime}-w^{\prime} \frac{\partial \bar{q}_{v}}{\partial z} \\
+\rho^{-1}\left(\rho \overline{q_{v}^{\prime} w^{\prime}}\right)_{z} \\
\frac{D^{\prime} q_{c}^{\prime}}{D t^{\prime}}=C_{d}^{\prime}-A_{r}^{\prime}-w^{\prime} \frac{\partial \bar{q}_{c}}{\partial z}+\rho^{-1}\left(\rho \overline{q_{c}^{\prime} w^{\prime}}\right)_{z} \\
\frac{D^{\prime} q_{r}^{\prime}}{D t^{\prime}}-\frac{1}{\rho} \frac{\partial}{\partial z}\left(\rho V_{T} q_{r}^{\prime}\right)=A_{r}^{\prime}-E_{r}^{\prime}-w^{\prime} \frac{\partial \bar{q}_{r}}{\partial z}+\rho^{-1}\left(\rho \overline{q_{r}^{\prime} w^{\prime}}\right)_{z} .
\end{gathered}
$$

Note that on the microscale time, we need to advect by both fluctuations as well as the horizontal mean, $\overline{\mathbf{u}}_{h}(\mathbf{X}$, $z, T, t)$, calculated by

$$
\frac{\partial \overline{\mathbf{u}}_{h}}{\partial t}=-\rho^{-1}\left(\rho \overline{\mathbf{u}_{h}^{\prime} w^{\prime}}\right)_{z} .
$$


This is the fast time-scale horizontal momentum in a column model type of setup. Similarly, all the fluctuating source terms $E_{r}^{\prime}, C_{d}^{\prime}$, and $A_{r}^{\prime}$ need to be evaluated utilizing $\bar{q}_{v}, \bar{q}_{c}, \bar{q}_{r}$, and $\bar{\theta}$ computed from the column model equations in (3.3) at the current fast time. All of the terms in (3.3) are extremely easy to calculate rapidly and are needed for the microscale equations in (3.11), which supply the necessary fluxes for computing the longer time-averaged equations on mesoscales in (3.7)-(3.10). If spatial periodicity is imposed in the microscale fluctuations, then the equations in (3.3), (3.5), and (3.7)-(3.11) constitute a systematic basis for a superparameterization strategy (Grabowski 2003, 2004) for linking $O(10 \mathrm{~km})$ microscales to $O(100 \mathrm{~km})$ mesoscales with turbulent fluxes calculated by averaging over the microscale spatial period. This is also potentially a very useful systematic strategy for NWP parameterization with $10-\mathrm{km}$ horizontal resolution, since this systematic formulation also provides a framework for other new strategies for closure modeling with 10-km resolution (Moncrieff and Liu 2006). In an actual numerical model, the equations for the mean in (3.3) and the fluctuations (3.11) on the microscale time scale can be combined into standard bulk microphysics equations with $\mathbf{X}, T$ regarded as frozen parameters. See the discussion below in section 5 .

\section{The link between MMD and mesoscale equatorial synoptic-scale dynamics}

The MMD models in (3.3), (3.5), and (3.7)-(3.11) operate on the multiple spatiotemporal scales in (2.1) and (2.4),

$$
\left(L_{m}, L_{M}, T_{m}, T_{M}\right)
$$

Furthermore the spatiotemporal mesoscales $L_{m}=$ $O(100 \mathrm{~km})$ and $T_{m}=O(2 \mathrm{~h})$ are appropriate for the propagation of organized squall-line clusters (Moncrieff 1992; Emanuel 1994; Pandya and Durran 1996; Houze 2004). There is the observed statistical selfsimilarity (Mapes et al. 2006) of organized tropical convection from the microscales to mesoscales to equatorial synoptic scales to planetary scales and a recent quantitative analytic multiscale framework for the selfsimilarity cascade from mesoscales and equatorial synoptic scales to intraseasonal/planetary scales (Majda 2007) that is consistent with this observed selfsimilarity. Thus, it is very interesting to see the fashion in which the MMD models with active moisture derived in section 3 might crudely link to the mesoscale equatorial synoptic-scale dynamic (MESD) models in the cascade of multiscale dynamics [see Fig. 1; Majda (2007), his section 3]. Such a quantitative link is also interesting for putting the original superparameterization strategies of Grabowski $(2001,2003,2004)$ on a firm analytic basis for possible further developments.

The multiscale MESD models operate on the equatorial synoptic spatiotemporal scales $L_{E}=L_{S} \approx$ $O(1500 \mathrm{~km}), T_{E} \approx O(8 \mathrm{~h})$, and the mesoscales $L_{M}=$ $\epsilon L_{S}=O(150 \mathrm{~km}), T_{M}=\epsilon T_{S}=O(1 \mathrm{~h})$ (Majda 2007); thus, the spatiotemporal scales for MMD with active moisture in (4.1) and MESD roughly satisfy the selfsimilar scaling relation

$$
\left(L_{m}, L_{M}, T_{m}, T_{M}\right)=\epsilon\left(L_{M}, L_{S}, T_{M}, T_{S}\right) .
$$

The MESD models require both the low Froude number and weak temperature gradient assumptions for the horizontal velocity $\mathbf{U}_{h}$, potential temperature $\Theta$, and pressure $P$,

$$
\mathbf{U}_{h}=\epsilon \mathbf{u}_{h}, \quad \Theta=\epsilon \theta, \quad \text { and } \quad P=\epsilon p,
$$

and also forcing varying on the spatiotemporal mesoscales with roughly compatible approximate strength

$$
\left[S_{\theta}\right]=30 \mathrm{~K} / 8 \mathrm{~h}, \quad\left[\mathbf{S}_{\mathbf{u}}\right]=5 \mathrm{~m} \mathrm{~s}^{-1} / 8 \mathrm{~h}
$$

[Majda (2007), his Eqs. (4) and (7)]. To link the MMD models with active moisture with the MESD models, it is necessary to establish that the dynamical behavior of the MMD models on the outer mesoscales, $L_{M}$ and $T_{M}$, automatically and self-consistently matches the behavior of the MESD models on the inner scale required in (4.3) and (4.4). This matching is established in a crude fashion in the remainder of this section and is not immediately obvious since the MMD models do not directly involve low Froude number dynamics.

From (3.7), (3.9), and (3.10), the coarse-grained mesoscale equations for horizontal momentum, potential temperature, and pressure arise at order $\epsilon$ and are given by

$$
\begin{aligned}
\frac{\partial \mathbf{u}_{h}}{\partial T} & =-\nabla_{h} p+\mathbf{S}_{\mathbf{u}}^{\prime} \\
\frac{\partial \theta}{\partial T}+N^{2} w_{1} & =S_{\theta}^{\prime} \\
p_{z} & =\theta+S_{q}^{\prime} \\
\operatorname{div}_{X} \mathbf{u}_{h}+\rho^{-1}\left(\rho w_{1}\right)_{z} & =0 .
\end{aligned}
$$

In (4.5), $\left.S_{q}^{\prime}=\bar{\epsilon}\left\langle\bar{q}_{v}\right\rangle-\left\langle\bar{q}_{r}\right\rangle-\rho^{-1}\left(\rho \overline{\left\langle w^{\prime 2}\right.}\right\rangle\right)_{z}$ arises from moisture sources and turbulent fluxes varying only on the coarse-grained spatiotemporal mesoscales $L_{M}$ and $T_{M}$ as derived above in section 3 with a similar definition for $\mathbf{S}_{\mathbf{u}}^{\prime}, S_{\theta}^{\prime}$ using (3.7) and (3.9). Introducing the moisture pressure $p_{q}^{\prime}$ satisfying $\left(p_{q}^{\prime}\right)_{z}=S_{q}^{\prime}$, the equations in (4.5) can be written equivalently as dynamics for the linear anelastic Boussinesq equations, 


$$
\begin{aligned}
\frac{\partial \mathbf{u}_{h}}{\partial T} & =-\nabla_{h} p+\left(\mathbf{S}_{\mathbf{u}}^{\prime}-\nabla_{h} p_{q}^{\prime}\right) \\
\frac{\partial \theta}{\partial T}+N^{2} w_{1} & =S_{\theta}^{\prime} \\
p_{z} & =\theta \\
\operatorname{div}_{X} \mathbf{u}_{h}+\rho^{-1}\left(\rho w_{1}\right)_{z} & =0 .
\end{aligned}
$$

Now, the forced linear anelastic equations in (4.6) operating on the outer spatiotemporal mesoscales for MMD with active moisture are exactly the same as those for MESD [Majda (2007), his Eq. (13)], operating on the same spatiotemporal mesoscales in accordance with (4.2) under the low Froude number and weak temperature gradient assumptions in (4.3). In this crude sense the dynamics on the shorter faster scales of MESD automatically and self-consistently matches MMD with active moisture on its longer, slower, coarse-grained spatiotemporal scales, which are roughly the same scales from (4.2). This is the crude dynamic self-similarity and matching overlap between MMD and MESD on mesoscales (Majda 2007; Barenblatt 2003). Also note that according to the nondimensional units for MMD discussed at the beginning of section 2, the original units for potential temperature forcing are $30 \mathrm{~K} / 15 \mathrm{~min}$; the asymptotic self-consistency on the spatiotemporal mesoscales derived in (3.5) automatically guarantees that $S_{\theta}^{\prime}$ has reduced forcing strength with units $\epsilon 30 \mathrm{~K} / 15 \mathrm{~min}$, which is roughly compatible with (4.4) for a somewhat smaller value of $\epsilon=1 / 32$. Similar remarks apply for $\mathbf{S}_{\mathbf{u}}^{\prime}$. Also, it is important to remember that the source terms defined on the right-hand side of (4.5) include nonlinear advection in the MMD model unlike MESD. This incompatibility arises because the shortest time scale associated with the MESD models is a horizontal gravity-wave time scale while the related behavior in the MMD models occurs on an advective time scale. The need for this somewhat smaller value of $\epsilon$ to match MMD and MESD arises because Majda and Klein (2003) noted that there are actually intermediate advective scales between these two regimes differing by $\epsilon^{1 / 2}$ in space and time; such effects can accumulate on the Charney inertial scale $O(1000 \mathrm{~km})$ where the planetary $\beta$-effect and advection are comparable (Majda and Klein 2003). For simplicity, this scale is ignored in the crude matching here and results in the slightly smaller value of $\epsilon$ (by a factor of $1 / 3$ ) required to achieve (4.4) on mesoscales. The actual physical implication of this subtle distinction is interesting but beyond the scope of the present paper; on the other hand, factors of $1 / 3$ might be considered insignificant in practical terms.

\section{Systematic strategies for superparameterization and concluding discussion}

As noted earlier, the multiscale formulation from microscales to mesoscales leads to a systematic superparameterization strategy that is potentially useful for NWP with $O(10 \mathrm{~km})$ grid spacing. For computational reasons it is convenient to combine the leading-order equations in (3.3) and (3.11) involving microscale temporal fluctuations into essentially a single moist anelastic system with bulk cloud microphysics,

$$
\begin{aligned}
\frac{D^{\prime} q_{v}}{D t^{\prime}} & =-C_{d}+E_{r} \\
\frac{D^{\prime} q_{c}}{D t^{\prime}} & =C_{d}-A_{r} \\
\frac{D^{\prime} q_{r}}{D t^{\prime}}-\frac{1}{\rho} \frac{\partial}{\partial z}\left(\rho V_{T} q_{r}\right) & =A_{r}-E_{r} \\
\frac{D^{\prime} \theta}{D t^{\prime}}+N^{2} w^{\prime} & =L \frac{\theta_{0}}{p_{0}}\left(C_{d}-E_{r}\right) \\
\frac{D^{\prime} \mathbf{u}_{h}}{D t^{\prime}} & =-\nabla_{h}^{\prime} p^{\prime} \\
\frac{D^{\prime} w^{\prime}}{D t^{\prime}} & =-p_{z}^{\prime}+\theta^{\prime}+\left(\bar{\epsilon} q_{v}^{\prime}-q_{r}^{\prime}-q_{c}^{\prime}\right) \\
& +\rho^{-1}\left(\rho \overline{w^{\prime 2}}\right)_{z} \\
\operatorname{div}^{\prime} \mathbf{u}_{h}^{\prime}+\rho^{-1}\left(\rho w^{\prime}\right)_{z} & =0
\end{aligned}
$$

and the larger mesoscale anelastic and hydrostatic constraints on the micro-time scale,

$$
\begin{aligned}
\operatorname{div}_{X} \overline{\mathbf{u}}_{h}+\rho^{-1}\left(\rho \bar{w}_{1}\right)_{z} & =0 \\
\bar{p}_{z} & =\bar{\theta}+\left(\bar{\epsilon} \bar{q}_{v}-\bar{q}_{r}-\epsilon \bar{q}_{c}\right)-\rho^{-1}\left(\rho \overline{w^{\prime 2}}\right)_{z} .
\end{aligned}
$$

The key point for (5.1) is that these equations are essentially the moist anelastic equations in the microscale variables $(x, t)$ alone with the mesoscale spatiotemporal variables $(X, T)$ entering as frozen parameters since $D^{\prime} / D t^{\prime}$ involves only small-scale advection; the only difference is that the constraint, $\overline{w^{\prime}}=0$, needs to be enforced at each vertical level and this is immediately satisfied through the second equation in (5.2). The large-scale vertical velocity $\bar{w}_{1}$ is calculated readily from vertical integration of the anelastic constraint in (5.2) with $\overline{\mathbf{u}}_{h}(\mathbf{X}, z, t)$ determined from (5.1). As in superparameterization (Grabowski 2004), the role of the system in (5.1) and (5.2) is to explicitly calculate the turbulent fluxes needed to drive the large-scale linear anelastic system in (3.7)-(3.10) involving the coarse- 
grained mesoscale spatiotemporal averages, $\left\langle\overline{\mathbf{u}}_{h}\right\rangle(\mathbf{X}, z$, $T),\langle\bar{\theta}\rangle(\mathbf{X}, z, T)$, etc. If spatial periodicity is imposed on (5.1) and (5.2) on the microscales with the period interval $L_{M} \approx 10 \mathrm{~km}$, then the spatial averages of the turbulent fluxes needed for (3.7)-(3.10) are readily calculated by periodic averaging in (2.7); computational efficiency in solving (5.1) and (5.2) is automatically gained because of the massively parallel character of solving (5.1) and (5.2) with the mesoscale variables frozen. In addition, dimensional reduction can be achieved for (5.1) and (5.2) by solving suitable problems involving a single horizontal dimension (Grabowski 2001, $2003,2004)$. In this fashion, the spatial averages for the fluxes required in (3.7)-(3.10) are achieved readily.

\section{a. A heterogeneous multiscale method}

The formulation in (5.1) and (5.2) for the microscales coupled with the coarse-grained equations in (3.7)(3.10) operating on larger, longer spatiotemporal scales can be viewed as a heterogeneous multiscale method (HMM) for mesoscale convection so that techniques from the applied mathematics literature (E and Engquist 2003) can be utilized further to reduce the computational labor due to the disparity in time scales. In the language of HMM, the microsolver is the equations in (5.1) and (5.2), which evolve on the rapid time scale, while the macrosolver is the equations in (3.7)-(3.10) for the coarse-grained spatiotemporal averages. Clearly, the rapidly varying equations in (5.1) and (5.2) require a small time step $\Delta t$, with $m \Delta t=T_{m} \approx 15 \mathrm{~min}$ while the macroscale equations in (3.7)-(3.10) allow a much bigger time step $\Delta T$ for comparable time resolution with $m \Delta T=T_{M} \approx 2.5 \mathrm{~h}$. For a given accuracy the ratio of the time step size $\Delta \mathrm{T} / \Delta t=T_{M} / T_{m} \approx 10$; thus, one can implement a predictor-corrector strategy as done by Grabowski (2004), where the values of the coarse-grained variables from (3.7) to (3.10) at a given large-scale time $K \Delta T$ are frozen while a loop of the massively parallel microsolver in (5.1) and (5.2) is utilized with the smaller time step, $\Delta T T_{m} / T_{M}=\Delta t$, in order to compute the update of the time averages needed in the macrosolver from (3.5) and (3.7)-(3.10). The equations in (3.7)-(3.10) are then updated by a standard solver on the coarse mesh although it is desirable to use a high-resolution solver to reduce numerical diffusion and dispersion on these large scales. Note that it is not necessary to use a hydrostatic anelastic equation to solve (3.7)-(3.10); the nonhydrostatic anelastic equations can be utilized with the balanced initial constraint, $\bar{w}=0$, and $w_{1}$ determined from (2.5), so that subtle issue in the numerical modeling of hydrostatic equations can be avoided (Orlanski 1981). A detailed implementation of the above computational strategy will be presented elsewhere in the near future with other collaborators. The same strategy can also be used with the fully compressible solvers for each piece.

\section{b. Generalized column modeling in NWP}

The other extreme in the present formulation is to replace the microscale equations in (5.1) and (5.2) by a parameterized form of the column model equations in (3.3) so that a detailed solution of (3.11) is completely neglected and (5.1) and (5.2) become a column model. The use by Moncrieff and Liu (2006) of the BettsMiller parameterization with added vertical dipole heat sources can be regarded as a special case of this extreme version of the present formulation. In fact, the present systematic setup can be utilized in a similar fashion to design stochastic parameterizations (Khouider et al. 2003) or simplified multicloud parameterizations with shear (Khouider and Majda 2006) for the microsolver in (5.1) instead of computationally intensive superparameterizations, while potentially capturing important physical effects beyond column modeling. The most high-resolution and expensive version of NWP involves detailed cloud-resolving modeling on a fine mesh with roughly 1-km resolution; the key new features with such high resolution are convective organization through moisture and orography (Moncrieff and Liu 2006) resulting from gravity-wave interaction. An approximation of the present flexible systematic approach, as noted above, has the potential to retain these attractive features statistically but with a much lower computational overhead. This is especially true when combined with the HMM numerical formulation. These possibilities are clearly a major issue for further research.

\section{c. Superparameterization and the equatorial synoptic scales}

The original superparameterization strategy (Grabowski 2001, 2003, 2004) utilized cloud-resolving models on the mesoscales involving a $200-\mathrm{km}$ periodic domain coupled with a coarse-mesh resolution, $\Delta x=1200$ $\mathrm{km}$, which is essentially the equatorial synoptic scale; thus, there is a scale gap between the mesoscales and planetary scales and the original superparameterization method cannot resolve tropical equatorial synopticscale variability such as convectively coupled Kelvin waves and two-day waves (Wheeler and Kiladis 1999). The theory developed in section 4, which demonstrates a self-consistent spatiotemporal overlap between the MMD and MESD models, gives strong analytic support that there is no inherent limitation in the formulation of superparameterization that limits the resolution of dy- 
namics on the equatorial synoptic scales; the results in section 4 strongly suggest that only higher spatial resolution on the coarse mesh is needed to resolve synopticscale dynamics. Of course, the computational labor in the superparameterization method increases under these circumstances.

Acknowledgments. The author thanks Rupert Klein for his constructive criticism of an earlier version of this manuscript. The author acknowledges generous support from the National Science Foundation under Grant NSF DMS-0456713 and the Office of Naval Research under Grant ONR N00014-05-1-0164.

\section{REFERENCES}

Barenblatt, G. I., 2003: Scaling. Cambridge University Press, 171 pp.

Biello, J. A., and A. J. Majda, 2005: A new multiscale model for the Madden-Julian oscillation. J. Atmos. Sci., 62, 1694-1721.

_ and - 2006: Modulating synoptic scale convective activity and boundary layer dissipation in the IPESD models of the Madden-Julian oscillation. Dyn. Atmos. Oceans, 42, 152 215 .

E, W., and B. Engquist, 2003: The heterogeneous multiscale methods. Comm. Math. Sci., 1, 87-132.

Emanuel, K. A., 1994: Atmospheric Convection. Oxford University Press, $580 \mathrm{pp}$.

Grabowski, W. W., 2001: Coupling cloud processes with the largescale dynamics using the cloud-resolving convection parameterization (CRCP). J. Atmos. Sci., 58, 978-997.

_, 2003: MJO-like coherent structures: Sensitivity simulations using the cloud-resolving convection parameterization (CRCP). J. Atmos. Sci., 60, 847-864.

_ 2004: An improved framework for superparameterization. $J$. Atmos. Sci., 61, 1940-1952.

- 2006: Comments on "Preliminary tests of multiscale modeling with a two-dimensional framework: Sensitivity to coupling methods." Mon. Wea. Rev., 134, 2021-2026.

—_, and P. K. Smolarkiewicz, 1996: Two-time-level semiLagrangian modeling of precipitating clouds. Mon. Wea. Rev., 124, 487-497.

— tropical convection in two-dimensional explicit numerical simulations. Quart. J. Roy. Meteor. Soc., 127, 445-468.

$\longrightarrow$, and 2004: Moisture-convection feedback in the Tropics. Quart. J. Roy. Meteor. Soc., 130, 3081-3104.

Houze, R. A., Jr., 2004: Mesoscale convective systems. Rev. Geophys., 42, RG4003, doi:10.1029/2004RG000150.

Jung, J.-H., and A. Arakawa, 2005: Preliminary tests of multiscale modeling with a two-dimensional framework: Sensitivity to coupling methods. Mon. Wea. Rev., 133, 649-662.

Khairoutdinov, M., D. Randall, and C. DeMott, 2005: Simulations of the atmospheric general circulation using a cloud-resolving model as a superparameterization of physical processes. $J$. Atmos. Sci., 62, 2136-2154.

Khouider, B., and A. J. Majda, 2006: A simple multicloud parameterization for convectively coupled tropical waves. Part I: Linear analysis. J. Atmos. Sci., 63, 1308-1323. $\longrightarrow,-$ and M. A. Katsoulakis, 2003: Coarse-grained stochastic models for tropical convection and climate. Proc. Natl. Acad. Sci. USA, 100, 11 941-11946.

Klein, R., 2000: Asymptotic analyses for atmospheric flows and the construction of asymptotically adaptive numerical methods. Z. Angew. Math. Mech., 80, 765-777.

_ 2004: An applied mathematical view of meteorological modeling. Applied Mathematics Entering the 21st Century: Invited Talks from the ICIAM 2003 Congress, J. M. Hill and R. Moore, Eds., Proceedings in Applied Mathematics, Vol. 116, SIAM, 227-289.

, and A. Majda, 2006: Systematic multiscale models for deep convection on mesoscales. Theor. Comput. Fluid Dyn., 20, $525-551$.

Lin, J.-L., and Coauthors, 2006: Tropical intraseasonal variability in 14 IPCC AR4 climate models. Part I: Convective signals. J. Climate, 19, 2665-2690.

Lipps, F. B., and R. S. Hemler, 1982: A scale analysis of deep moist convection and some related numerical calculations. $J$. Atmos. Sci., 39, 2192-2210.

Majda, A. J., 2003: Introduction to PDEs and waves for the atmosphere and ocean. Courant Lecture Notes in Mathematics, Vol. 9, American Mathematical Society, 234 pp.

_ 2007: New multiscale models and self-similarity in tropical convection. J. Atmos. Sci., 64, 1393-1404.

— Tropics. J. Atmos. Sci., 60, 393-408.

- and J. A. Biello, 2004: A multiscale model for the intraseasonal oscillation. Proc. Natl. Acad. Sci. USA, 101, 4736-4741.

— , R. V. Abramov, and M. J. Grote, 2005: Information Theory and Stochastics for Multiscale Nonlinear Systems. CRM Monograph Series, Vol. 25, Amer. Math. Soc., 133 pp.

Mapes, B. E., S. Tulich, J.-L. Lin, and P. Zuidema, 2006: The mesoscale convection life cycle: Building block or prototype for large-scale tropical waves? Dyn. Atmos. Oceans, 42, 3-29.

Moncrieff, M. W., 1992: Organized convective systems: Archetypal dynamical models, mass and momentum flux theory, and parameterization. Quart. J. Roy. Meteor. Soc., 118, 819850.

— , and C. Liu, 2006: Representing convective organization in prediction models by a hybrid strategy. J. Atmos. Sci., 63, 3404-3420

Orlanski, I., 1975: A rational subdivision of scales for atmospheric processes. Bull. Amer. Meteor. Soc., 56, 527-530.

_ 1981: The quasi-hydrostatic approximation. J. Atmos. Sci., 38, $572-582$.

Pandya, R. E., and D. R. Durran, 1996: The influence of convectively generated thermal forcing on the mesoscale circulation around squall lines. J. Atmos. Sci., 53, 2924-2951.

Randall, D., M. Khairoutdinov, A. Arakawa, and W. Grabowski, 2003: Breaking the cloud parameterization deadlock. Bull. Amer. Meteor. Soc., 84, 1547-1564.

Schmidt, H., and R. Klein, 2003: A generalized level-set/in-cellreconstruction approach for accelerating turbulent premixed flames. Combust. Theor. Modell., 7, 243-267.

Slingo, J. M., and Coauthors, 1996: Intraseasonal oscillations in 15 atmospheric general circulation models: Results from an AMIP diagnostic subproject. Climate Dyn., 12, 325-357.

Wheeler, M., and G. N. Kiladis, 1999: Convectively coupled equatorial waves: Analysis of clouds and temperature in the wavenumber-frequency domain. J. Atmos. Sci., 56, 374-399. 